\title{
The Qualitative Electrochemical Determination of Multiple Components in Seaweed Fertilizer
}

\author{
Xinyi Zhang ${ }^{1}$, Jiakun Zhou ${ }^{1}$, Zemin Li $^{1}$, Yimin Qin ${ }^{2 *}$, Ruitao Yu ${ }^{3,4}$, Huaiwei Zhang ${ }^{1}$, Yuhong Zheng ${ }^{5}$, \\ Jiangwei Zhu ${ }^{6}$, Demeng Zhang ${ }^{2 *}$ and $\mathrm{Li} \mathrm{Fu} \mathrm{u}^{1,2 *}$ \\ ${ }^{1}$ College of Materials and Environmental Engineering, Hangzhou Dianzi University, Hangzhou, PR \\ China \\ ${ }^{2}$ Ministry of Agriculture Key Laboratory of Seaweed Fertilizers, Qingdao Brightmoon Seaweed \\ Group Co Ltd, Qingdao, PR China \\ ${ }^{3}$ Qinghai Key Laboratory of Tibetan Medicine, Northwest Institute of Plateau Biolog, Chinese \\ Academy of Sciences, Xining, China \\ ${ }^{4}$ Key Laboratory of Tibetan Medicine Research, Northwest Institute of Plateau Biology, Chinese \\ Academy of Sciences, Xining, PR China \\ ${ }^{5}$ Institute of Botany, Jiangsu Province and Chinese Academy of Sciences (Nanjing Botanical Garden \\ Mem. Sun Yat-Sen), Nanjing 210014, P.R. China \\ ${ }^{6}$ Collaborative Innovation Center of Sustainable Forestry in Southern China of Jiangsu Province, \\ (Nanjing Forestry University), Nanjing 210037, China \\ *E-mail: yiminqin1965@126.com, zdm@ bmsg.com, fuli@hdu.edu.cn
}

doi: $10.20964 / 2019.07 .16$

Received: 7 March 2019 / Accepted: 24 April 2019 / Published: 10 June 2019

Seaweed fertilizer refers to fertilizer created by extracting the active ingredients from raw seaweed that is then applied to plants as a nutrient source to promote growth and increase yield. A quick method of analysing seaweed fertilizer quality has practical value. In this work, we established a simple electrochemical method for analysing commercial seaweed fertilizer by comparing the contributions of each electro-active ingredient. The electrochemical profiles of 3-hydroxyphenylacetic acid, gibberellin, lycine, abscisic acid, indole-3-acetic acid and zeatin were first recorded in a database. Then, the electrochemical profiles of four commercial seaweed fertilizers were recorded and examined to analyse the contribution of each ingredient.

Keywords: Seaweed fertilizer; Electrochemical analysis; Differential pulse voltammetry; Electrochemical sensor; Fingerprint 
(C) 2019 The Authors. Published by ESG (www.electrochemsci.org). This article is an open access article distributed under the terms and conditions of the Creative Commons Attribution license (http://creativecommons.org/licenses/by/4.0/). 